\title{
LE LÉMAN COMME FRONTIÈRE
}

\author{
Par Charles Biermann
}

Avec deux illustrations

La frontière entre la France, plus précisément la province de Savoie et le département de Haute-Savoie, et la Suisse, en l'espèce le canton de Vaud, passe par le Léman. C'est un lac, c'est-à-dire une étendue d'eau moins grande qu'une mer, dans le cas particulier un des plus grands lacs d'Europe, le plus vaste de ceux de l'Europe occidentale; il se trouve sur le parcours du Rhône, qui s'y jette près de Villeneuve, qui en sort à Genève, et peut être considéré comme une section de ce fleuve.

Doit-il, au point de vue du tracé de la frontière, être traité comme un fleuve ou comme une mer? On agit en effet différemment à leur égard. Sur un fleuve, la frontière suit le milieu de son cours, et s'il se subdivise en deux ou plusieurs bras, le milieu du courant principal. C'est ainsi que court, le long du Rhin entre Bâle et Lauterbourg, la frontière franco-allemande dès 1919. Sur une côte maritime, sous le nom d'eaux territoriales, on comprend encore dans le territoire national une bande d'eau de 3 milles marins de large, c'est-à-dire environ $5 \mathrm{~km} 555 \mathrm{~m}$. Le Léman, avec ses $13 \mathrm{~km}$ de largeur dans la plus grande partie du Grand lac, laisserait une zone neutre ou internationale de $2 \mathrm{~km}$ entre les eaux françaises et suisses. Il est vrai que cette zone, étroite et fermée de tous côtés, n'aurait pas l'utilité qu'elle a sur mer, où elle constitue la voie internationale accessible à tous les pavillons.

Il est donc indiqué de traiter un lac, malgré sa largeur, comme une rivière plutôt que comme une mer. Cependant, à l'autre bout de la Suisse, un autre lac, celui de Constance, se présente autrement encore. Aucun traité n'y a fixé les limites de la Suisse, de l'Autriche, de l'Allemagne, ni des trois Etats allemands riverains, la Bavière, le Wurtemberg et Bade. Aussi les juristes allemands considéraient-ils ce lac, ou tout au moins la partie en amont de Constance, le lac supérieur (Obersee), comme un territoire indivis, un condominium, appartenant collectivement aux cinq Etats. La Suisse n'a jamais accepté cette théorie, qui paraît être abandonnée aujourd'hui.

Pour ce qui en est du Léman, l'hésitation n'existe pas. Le traité de Lausanne du 30 octobre 1564, conclu entre LL.EE. de Berne et le duc de Savoie, a fixé au milieu du lac la frontière entre les deux contractants. Jusqu'alors, et depuis 1536, les Bernois ayant conquis, en même temps que le Pays de Vaud, le Chablais occidental, et les Valaisans s'étant avancés de leur côté jusqu'à la Dranse de Thonon, le Léman avait été un lac suisse. Avant 1536, c'était un lac savoyard, depuis que les comtes de Savoie, maîtres de la rive méridionale, avaient pris pied sur la rive nord, et d'abord à Morges, où ils fondèrent successivement le château et la ville, vers 1290 , puis sur la plus grande partie du Pays de Vaud. Auparavant, au XIIIe siècle et antérieurement, il n'y avait d'un côté du lac comme de l'autre, au nord comme au sud, qu'une mosaĩque d'Etats féodaux, à la fois petits et instables, attachés à la personnalité de leurs princes, s'accroissant avec les puissants, se réduisant, s'effondrant même avec les faibles. Pourtant, dès cette époque, on voit apparaître la notion de frontière lacustre dans des actes d'hommage, des reconnaissances de droits ou des chartes de franchises. F. A. Forel, dans Le Léman (III, p. 506), en a indiqué un certain nombre. Chaque fois les limites du territoire sont établies au milieu du lac. Le traité de Lausanne n'a fait donc que reprendre d'anciennes pratiques en s'exprimant comme suit à son article 20: que le milieu du lac, aux endroits des abordantes terres et seigneuries doive être et demeurer la ferme, vraie, clarifiée et nommée limite sur le dit lac...

Lorsqu'un traité prévoit des changements territoriaux, il est d'usage de charger une commission mixte de les préciser sur le terrain. Rien n'a été fait après 1564 . Bien plus ni le traité de Thonon, du 3 mars 1569, qui rendit à Emmanuel-Philibert le Chablais oriental, occupé par les Valaisans, ni celui de Turin, de 1816, qui concéda à Genève plusieurs communes sardes, n'ont fixé la ligne de raccordement entre la tive à St-Gingolph ou Hermance et le milieu du lac. C'est pourquoi le Conseil fédéral suisse a désigné en 1931 une délégation chargée d'entrer en pourparlers avec les représentants français en vue de déterminer la frontière franco-suisse dans le Léman. Ces pourparlers on été interrompus par la guerre en 1939 avant d'avoir mené à une entente définitive. Ils n'ont pas encore été repris. 


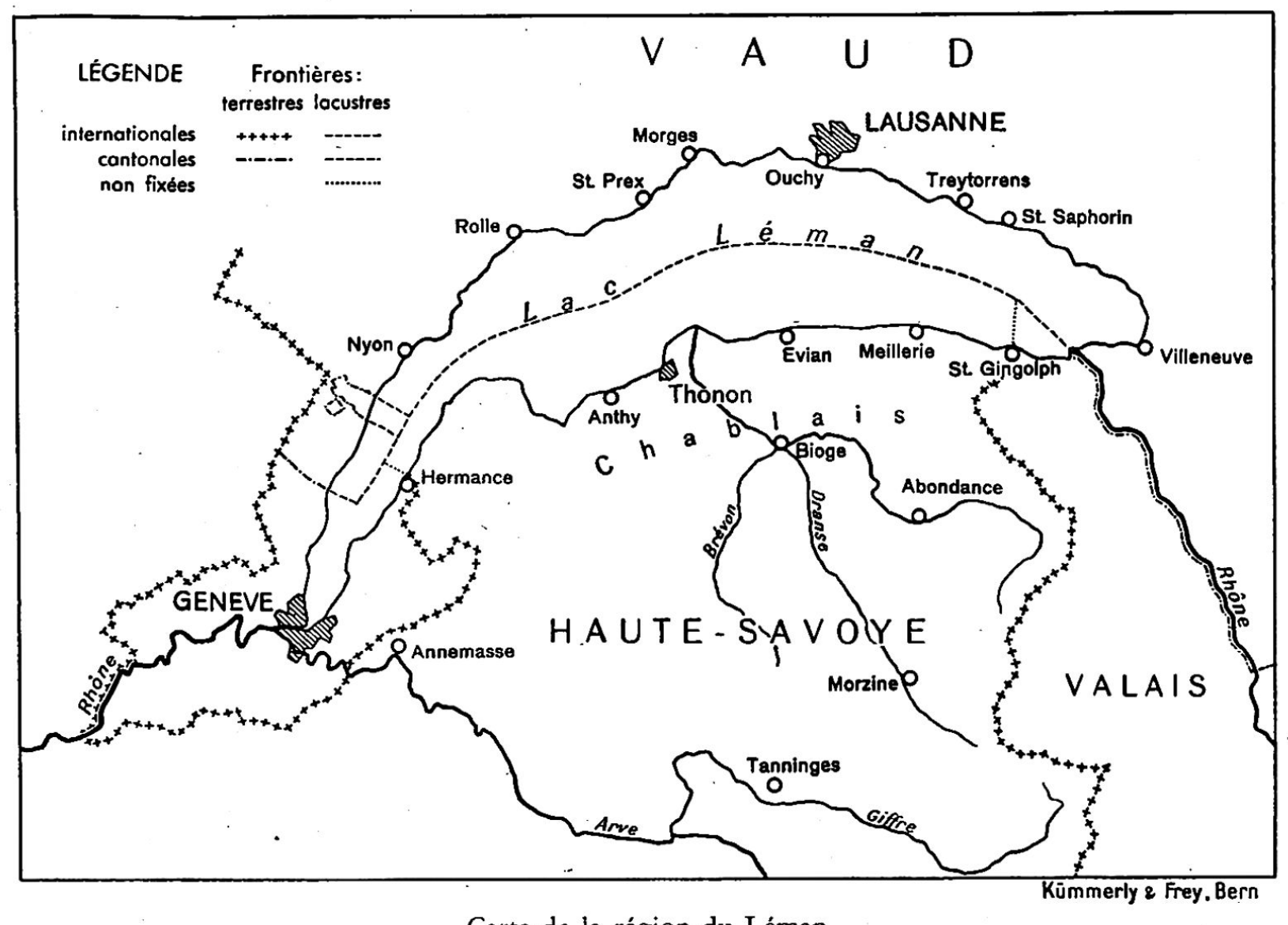

Sur le papier, une grande partie de la Haute-Savoie fut comprise de 1816 à 1919 dans la neutralité suisse, avec droit pour la Suisse de l'occuper militairement en cas de conflit. La Suisse rappela son droit en 1859, 1870 et 1914, mais sans en user. Pendant un siècle donc, le Léman fut un lac neutre. Le traité de Versailles y mit fin, la situation n'étant plus la même depuis 1860, où la Savoie est devenue française, que lorsqu'elle faisait partie des Etats sardes, où sa position excentrique en rendait la défense difficile.

Un lac aussi grand que le Léman n'est pas seulement une frontière; c'est aussi une voie de communication, d'autant plus précieuse que les routes terrestres ont encore besoin d'améliorations. Dès l'époque romaine, nous voyons des corporations de nautoniers à Genève et Lausanne. A l'aube de son indépendance, Genève n'eut pas d'autre moyen de communiquer avec ses alliés de Berne et Fribourg que par le lac. Le port de Morges était le point d'embarquement pour Genève des marchandises venues du Nord, celui d'Ouchy recevait le trafic d'Italie par le Simplon ou le Grand St-Bernard. Ce n'est qu'à la fin du XVIIIe siècle qu'un "grand chemin» fut construit de Berne à Genève par Lausanne, dont les pierres milliaires sont encore visibles et dont la route actuelle suit le tracé. C'est plus tard encore que fut aménagée la route de Lausanne à Villeneuve, qui n'était jusque là qu'un mauvais chemin de vignes.

Jusqu'à quel point la navigation fut-elle libre sur ce lac frontière? Cela dépendit des époques. Au temps où le duc de Savoie nourrissait des idées de conquête ou des sentiments de revanche, sa flotte de guerre devenait plus entreprenante et les routes d'eau se fermaient à ses adversaires. Si le traité de Lausanne consacre l'abandon définitif de ses droits sur le Pays de Vaud, le duc ne renonce pas à l'espoir de recouvrer Genève; et ses tentatives dans cette direction déclenchent des mouvements des troupes bernoises en faveur de leurs combourgeois, des incursions même jusque dans la Savoie du Nord. Il ne s'agit pas seulement des contestations entre Savoyards et Suisses, mais aussi entre Savoie et France. A plusieurs reprises, sous Louis XIII, sous Louis XIV, la Savoie fut partiellement ou totalement occupée par les armées françaises, et de 
facto, sinon de jure, le lac ne séparait plus les deux signataires du traité qui en avait fait une frontière. Ajoutons à ces causes l'hostilité naturelle, en ces siècles d'intolérance, entre gens de confession différente, catholique sur la rive sud, protestante sur celle du nord. Forel donne, dans Le Léman, une liste des rencontres, signalées par les documents, entre bateaux ennemis, grandes barques de guerre, galères ou brigantins, et vaisseaux de commerce. Ces engagements sont surtout nombreux au XVIe et au XVIIe siècles. Ils devinrent ensuite moins fréquents, en prenant au XVIIIe siècle le caractère d'actes de piraterie, comme le montre l'aventure du banneret Blanchet, de Lutry, en 1706.

$\therefore$, Ces attaques ont laissé dans la tradition orale un souvenir qui s'est conservé jusqu'à nos jours sur la rive vaudoise. A St-Saphorin, le quartier du bord du lac est appelé encore le quartier savoyard; la grande route qui le sépare maintenant du reste du village a interrompu la communication qui existait entre les étages supérieurs des maisons, pour permettre aux habitants de s'enfuir en cas de débarquement subit des Sâvoyards. A Treytorrens (Puidoux), le bâtiment le plus proche de l'eau, de grandes dimensions, possède des caves voûtées, au niveau inférieur du sol en forte pente; c'est là qu'on aurait remisé les barques «au temps des pirates». Relevons, sur cette côte, le très petit nombre de localités directement à la rive, et toutes celles qui avaient cette position étaient de petites villes ou des bourgs fortifiés.

A partir du XIXe siècle, les privilèges et les interdictions, comme les sentiments d'hostilité, ont disparu et la navigation a pu se faire sur le Léman en toute liberté. Elle a été un certain temps très active, surtout lorsque, après l'annexion à la France, en 1860, la Savoie du Nord fut devenue zone franche. Des carrières de Meillerie; qui bordent le lac, de grandes barques aux voiles latines apportent la pierre de construction aux villes de Genève et de Lausanne en mal d'agrandissement. Les bateaux à vapeur complètent leurs services. La Compagnie générale de navigation sur le lac Léman, qui résulte de la fusion des premières entreprises de navigation à vapeur, étend son activité aux ports savoyards comme à ceux de la Suisse. Les relations entre Suisse et Savoie deviennent journalières. On voit même des maraîchers et des paysans de la région d'Evian contribuer, au début de ce siècle, à l'approvisionnement de Lausanne en légumes, et retenir, pour les marchés bi-hebdomadaires, leur place à l'année; il en vient de Neuvecelle, de Lugrin, de Maxilly, et même de Larringes, à $400 \mathrm{~m}$ au-dessus du niveau du lac. Il arrive parfois que c'est du beurre qui passe le lac à Evian.

Puis les courants de circulation se détournent de la traversée du lac. Les chemins de fer, qui longent les deux rives; accaparent peu à peu le trafic. On renonce à Lausanne au calcaire de Meillerie qu'on remplace par celui d'Arvel, près de Villeneuve, venu par voie ferrée. La Compagnie générale de navigation n'a plus guère pour clients que des touristes, et elle arrange ses horaires en conséquence.

La guerre, qui fait de la frontière une réalité, supprime momentanément toutes les relations entre les deux rives. Le traité de Versailles les réduit sensiblement, en mettant fin à la zone franche de l'annexion. La reprise du trafic des bateaux à vapeur, en 1945, n’a pas été complète. Six ports français seuls sont desservis: Evian, Thonon et les ports en face de Nyon. Ni Meillerie, ni Tourronde, ni Anthy ne sont touchés, pas plus d'ailleurs que St-Gingolph (France), cela pour des raisons de police.

Il y a cependant une organisation qui reste commune à tous les riverains, c'est la Société de Sauvetage du lac Léman (S.S.L.L.), société internationale sans en porter le nom. Fondée en 1885, elle réunit 33 sections, dont 7 françaises et 26 suisses. Inspirée de l'exemple de la Société centrale de Sauvetage des Naufragés à Paris, devenue à la fois sa marraine et sa bienfaitrice, elle a créé en faveur de ses membres une caisse de Secours mutuels en cas de maladie et en faveur des membres des équipes de secours une Caisse d'assurance-accidents. Et il y a lieu de remarquer que la dernière des sections fondées, reçue dans la société en 1948, est une section française, celle d'Anthy-Séchex. 
Parmi les navigateurs en risque de naufrage, il y a eu parfois des pêcheurs. Le Léman est en effet non seulement une frontière, non seulement une voie de transport, mais encore un atélier de travail. Le droit de pêche, étant à l'origine un droit seigneurial, n'a été abandonné par l'autorité aux simples particuliers que sous certaines conditions: restrictions dans les périodes de pêche, en vue de permettre le repeuplement spontané, dans l'emploi des engins, pour épargner le jeune poisson, dans le nombre des pêcheurs, soumis au paiement d'un permis. Jusqu'en 1880, chacun des Etats riverains avait sa législation propre. Cette année-là fut signée une convention internationale, qui fut renouvelée en 1900, mais dénoncée en 1911 par le canton de Vaud: les pêcheurs vaudois se plaignaient en effet des incursions des pêcheurs savoyards dans les eaux suisses et de la non-application des dispositions de la convention par les autorités françaises. Dès lors un concordat reproduisant dans les grandes lignes l'accord international, a été conclu entre les trois cantons suisses. Une nouvelle convention a été signée à Paris en 1924; ratifiée par les Chambres suisses, elle ne l'a pas été par celles de France. Toutefois il semblerait que le sous-préfet de Thonon serait disposé à appliquer dans les eaux françaises les principes adoptés par les cantons suisses riverains.

Ce n'est pas que la pêche soit aussi rémunératrice dans le Léman que dans les autres lacs subalpins. Du moins à l'époque actuelle. Car le Léman était originairement comme les autres un lac à corégones, où ce genre intéressant était représenté par la féra et la gravenche. Peu à peu, et cela surtout à la fin du XIXe et au début du XXe siècle, les corégones cèdent le premier rang aux perches. Les statistiques publiées par F. A. ForeL dans le IIIe volume de son Léman, le montrent clairement: la féra est expédiée de Savoie en Suisse en quantités qui décroissent de $83699 \mathrm{~kg}$ en 1897 à $20683 \mathrm{~kg}$ en 1902; pendant la même période, les exportations de perches passent de $4272 \mathrm{~kg}$ à $18169 \mathrm{~kg}$ (p. 646). Les achats des frères Lugrin, grands marchands de poissons à Genève, passent de $115000 \mathrm{~kg}$ en 1889 à $17000 \mathrm{~kg}$ en 1902 pour la féra, de $22000 \mathrm{~kg}$ à $54100 \mathrm{~kg}$ pour les perches, les lottes et les brochets (p. 649). A l'heure qu'il est, les corégones ne fournissent que le $17 \%$ de la pêche dans le Léman, au lieu de $71 \%$ dans les autres lacs suisses, tandis que les proportions correspondantes pour les perches sont $60 \%$ et $21 \%$.

Les causes de cette transformation sont à chercher soit dans la multiplication des pêcheurs, soit dans l'emploi de filets destructeurs. Parmi ces derniers, le pic a été inventé en 1888 par un pêcheur de Meillerie, qui en a obtenu des résultats si remarquables qu'il a été imité peu à peu sur tout le lac, et cette pêche de la féra a été poussée avec une telle intensité que le prix de ce poisson en était tombé rapidement de moitié. Avec la réduction du nombre des féras, les perches, qui ont leur habitat normal dans la zone littorale, ont pu s'introduire dans la zone pélagique, domaine des corégones, et y causer par leur voracité de grands ravages dans les banes de jeunes poissons. Quant aux pêcheurs, on estime qu'il y en a autant et même plus dans la zone française, malgré sa plus faible étendue ( $42 \%$ du total), que dans la zone suisse, où un recensement opéré l'hiver dernier en a constaté 290, dont 134 pêcheurs professionnels, 47 pêcheurs exerçant une autre occupation à titre secondaire et 109 pour qui la pêche n'est qu'une profession accessoire. En 1945, Vaud et Valais ont décidé de ne plus accorder de permis de pêche au filet qu'à des professionnels.

Enfin il faut reconnaître que la surveillance de la pêche est moins bien organisée en Savoie, où elle est concentrée à Thonon, qu'en Suisse, où les garde-pêche sont répartis sur tout le littoral.

Il existe, dans la région du Léman, une autre communauté de travail que celle de la pêche; c'est celle qui se forme entre montagnards et gens de la plaine, plus précisément ceux du vignoble. Ces deux éléments complémentaires, la culture exigeante, mais rémunératrice qu'est la vigne, et la simplicité de la vie pastorale, se trouvent ici séparés par le Léman, qui ne réussit pourtant pas à étouffer l'appel de main-d'œuvre lancé par les vignerons des côteaux vaudois et auquel le Chablais répond, m'assure-t-on, depuis 


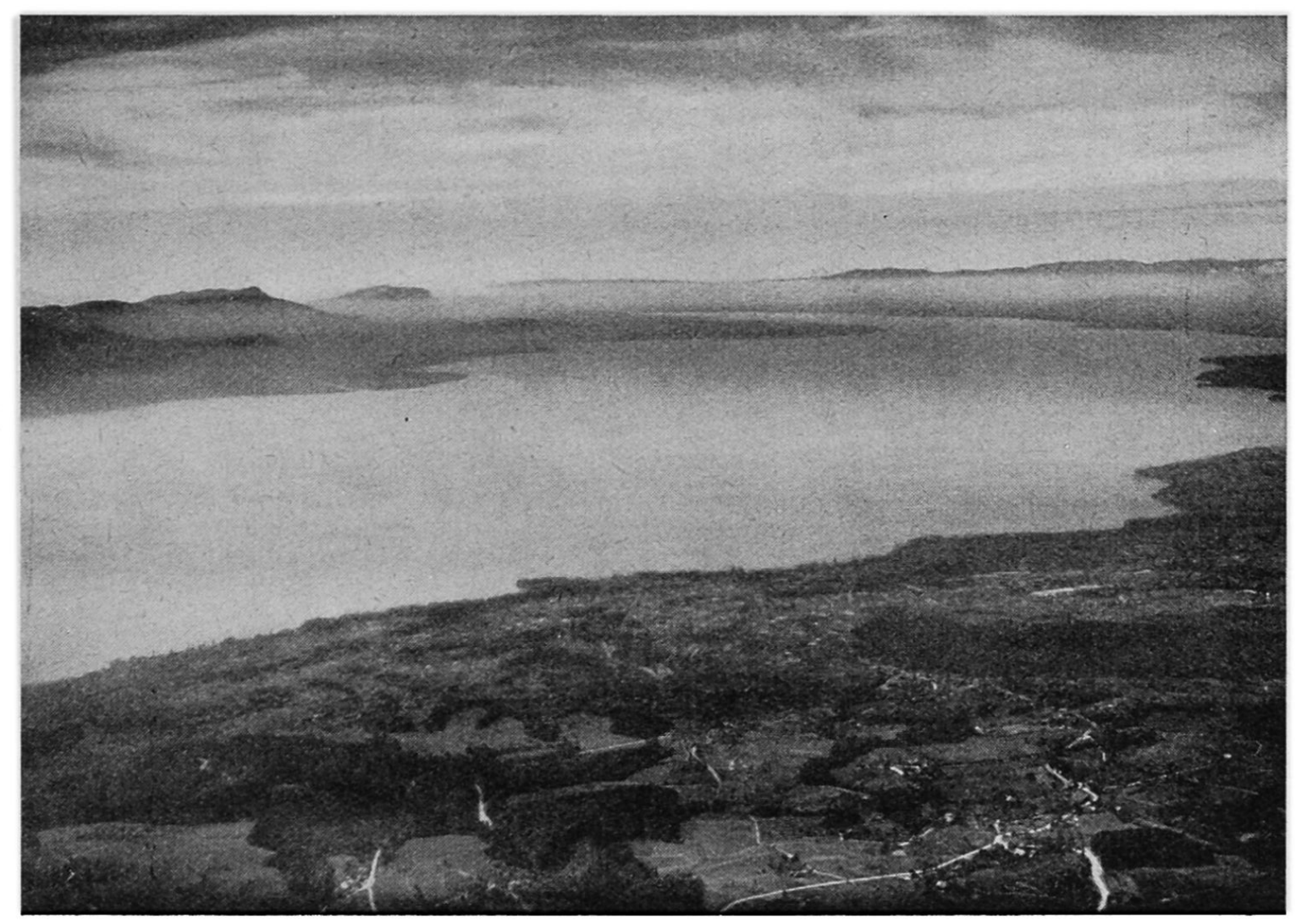

Le Léman vu des environs de Lausanne. (Photo Swissarr)

au moins un siècle, c'est-à-dire antérieurement à la constitution de la zone franche, antérieurement à la dépréciation de la monnaie française par rapport à celle de Suisse, antérieurement aux destructions dues à la deuxième guerre mondiale. Primitivement les travailleurs savoyards auraient appartenu à l'un et l'autre sexe: les hommes se seraient engagés pour le labour, en quoi ils sont maintenant remplacés par les machines; les femmes furent, et sont encore maintenant, suivant un terme local, des effeuilleuses, c'est-à-dire qu'elles sont chargées de l'épamprage et de l'ébourgeonnement, ainsi que de l'attache des sarments conservés. Ce travail leur prend environ trois semaines, en général au mois de mai ou de juin, temps pendant lequel elles sont les hôtes de celui qui les a engagées. Outre un salaire en espèces, les effeuilleuses sont logées et nourries, et les maisons vigneronnes de la rive vaudoise leur réservent toutes une ou plusieurs chambres, dénommées chambres des Savoyardes. L'engagement se renouvelait autrefois année après année, les patrons se succédaient de père en fils, les ouvrières de mère à fille ou à belle-fille ou à nièce. La dernière guerre, où la Savoie a été occupée, a brisé ces relations; elles ont été interrompues quelques années. Quand il a été possible de les renouer, il fallut se soumettre aux prescriptions de la police, soucieuse de surveiller le séjour des travailleuses étrangères. Tandis que celles-ci gagnaient autrefois le port du Léman le plus rapproché, pour rejoindre leur lieu de destination, elles ont dû, dès 1945 , renoncer à la voie d'eau et se détourner par l'une ou l'autre des extrémités du lac, où les autorités suisses avaient installé des postes d'entrée, à Moillesullaz, en face d'Annemasse, et à St-Gingolph. C'est à cette circonstance que l'on doit de voir le recrutement de cette main-d'œuvre se faire à Annemasse même, puis dans la vallée du Giffre, à Tanninges, à Verchaix, alors que jusqu'ici il était restreint au Chablais. Cependant, c'est bien là, sur les hauteurs au-dessus d'Evian et de Thonon, et surtout dans 
les trois vallées de la Dranse d'Abondance, de la Dranse de Morzine et du Brévon, qui confluent à Bioge pour tendre au Léman en un seul sillon, que résident la plupart des effeuilleuses venues en 1945 dans le vignoble vaudois. Si les relevés de police nous fournissent des renseignements que seule une longue enquête aurait pu nous livrer autrefois, il ne faut donc les utiliser qu'avec précaution, et ce serait encore plus le cas pour ceux des années suivantes, où le vignoble neuchâtelois et celui du lac de Bienne ont aussi recouru, la main-d'œuvre jurassienne faisant défaut, aux effeuilleuses savoyardes.

Le Léman est une frontière, c'est ce que les hommes ont décidé; la nature en avait fait plutôt un trait d'union entre les populations de ses deux rives, trait d'union qui tend à se reconstituer chaque fois que la situation est normale, pour se rompre dans les périodes troublées.

Cette note a été rédigée à l'aide des ouvrages signalés, notamment F. A. Forel: Le Léman, 3 vol.; elle s'appuie, en outre, sur une enquête faite auprès de $1^{\circ}$ Service topographique du Département militaire fédéral (lettre du 17 juin 1948). $-2^{\circ}$ Direction du Ve arrondissement des Douanes suisses (lettre du 23 juin 1948, accompagnée du Statut juridique du lac Léman - Liste polycopiée des traités et conventions qui le règlent). $-3^{\circ}$ Office cantonal (vaudois) de contrôle des habitants et de la police - des étrangers (lettre du 12 juin 1948). - $4^{\circ}$ Service de la Viticulture du Département (vaudois) de l'Agriculture, de l'Industrie et du Commerce (fiches d'engagement des effeuilleuses savoyardes). $5^{\circ}$ Serviçe des. Forêts, Chasse et Pêche du Département (vaudois) de l'Agriculture, de l'Industrie et du Commerce (lettre du 20 juin 1948, accompagnée du tableau du recensement des pêcheurs suisses du lac Léman effectué en 1947/1948 par l'inspection fédérale de la pêche). - $6^{\circ}$ Société de Sauvetage du lac Léman (lettre de son secrétaire général du 18 juin 1948).

\section{LA FRONTIERA DEL LEMANO}

La frontiera del Lemano non è stata fissata sufficientamente, dai trattati di confine. Prima assai importante per la navigazione e la pesca, essa perdette quest'importanza dopo la prima guerra mondiale, senza però impedire la continuazione delle relazioni economiche, specie la richiesta d'operai della Savoia per il Cantone di Vaud.

\section{DER GENFERSEE ALS GRENZE}

Die durch Staatsverträge nur ungenügend fixierte politische Grenze im Genfersee hatte früher erhebliche Bedeutung für Schiffahrt und Fischerei. Diese Bedeutung ist seit dem ersten Weltkrieg zurückgegangen. Aber die Grenze verhinderte auch sonst nie die wirtschaftlichen Beziehungen zwischen den Ländern, die sie trennt, insbesondere nicht die Wanderung der begehrten savoyischen Handwerker ins Waadtland.

\section{DIE LANDSCHAFT VON CHUR UND UMGEBUNG*}

\section{Von HANS BRUNNer}

Mit 3 Illustrationen

Ein herrlicher Herbstmorgen sieht unsere Gesellschaft von der Straße ins Bündner Oberland zwei Kilometer westlich der Stadt auf der ersten Schuttkegelwölbung ins taunasse Gras treten, um von diesem günstigsten Standort aus einen Überblick über die nähere und weitere Umgebung von Chur zu gewinnen.

Die Landschaft von Chur ist im weitern Sinne das Churer Rheintal von der Mündung der. Landquart bis zum Zusammenfluß der beiden Rheine bei Reichenau. Sie hat trotz der markanten Biegung aus dem Längs- ins Quertal bei Chur einen einheitlichen, einfachen und großzügigen Aufbau. Rechtsseitig bilden die teilweise ruinenhaften Formen, der Hochwanggruppe vom Montalin bei Chur bis zum Gü am Ende des niedrigen Valzeiner Grates den Talrahmen. Von Chur an übernimmt in WSW-Richtung der breite Fuß der Dreibündensteingruppe die gleiche Rolle. Gestein und Landschafts-

* Bericht zur Exkursion der Naturwissenschafter und Geographen anläßlich der Tagung der Schweiz. Gymnasiallehrer vom 9./10. Oktober 1948 in Chur. Leitung: Dr. J. NiEderer und Hs. BrunNER. 